\title{
Word coding: The role of intrinsic and extrinsic features*
}

\author{
DOUGLAS L. NELSON \\ University of South Florida, Tampa, Fla. 33620
}

The purpose of this experiment was to investigate the effect of extrinsic cues in processing words. In a serial anticipation task, starting position was constant or varied and the stimulus words were phonetically distinct, highly similar, or highly similar and arranged into a rhyming pattern. Reliance upon external features, temporal constancy cues, was greater when items were phonetically similar than when distinct. Furthermore, rhyming pattern served as an effective external cue, even when practice did not begin on the first item of the sequence on successive trials.

Words are represented in memory as complexes of distinctive features. This hypothesis represents the principal assumption of recent conceptualizations of word coding processes (e.g., Anisfeld \& Knapp, 1968; Nelson, 1972; Underwood, 1969; Wickens, 1970). The features are usually identified as orthographic, phonetic, associative, semantic, and imaginal. According to this view, the process of coding a word essentially consists of priming one or more of its features within the context of the current learning task. Furthermore, Nelson (1972) has assumed that information concerning factors external to the word, or environmental features, may be coded in addition to its intrinsic attributes. For example, Nelson, Wheeler, \& Bercov (1970) manipulated stimulus meaningfulness and similarity among items learned by serial anticipation. Temporal constancy cues were or were not eliminated. In the constant conditions, item sequence and the temporal position of the items were confounded as in the standard procedure. Practice was started on the same item from trial to trial. In the varied conditions, item sequence was maintained but each trial was started on a different item in the list. The findings indicated that, as distinctive semantic-associative and phonetic features decreased, there was a proportionately greater reliance upon positional or environmental cues. When meaningfulness was low and items were also phonetically similar, $75 \%$ of the Ss showed little or no progress in learning the list if these cues were eliminated, even after 100 trials of practice. Disruption of the position cues interfered with recall even when the stimuli were highly differentiated English words. Thus, relative position information may represent an important distinguishing characteristic of words coded in a serial anticipation task.

The aim of the present experiment was to continue the investigation of the relative functional effectiveness

*Supported by Grant MH 16360 to the author from the National Institute of Mental Health. Appreciation is expressed to Barbara Mikelait and Marcia Folino for collecting the data of the experiment. of extrinsic cues in coding words. These cues were manipulated by varying starting position and phonetic pattern within sets of words coded in a serial anticipation task. Thus, starting position was either constant or varied on successive trials. Formal similarity among the stimulus words was low or high and, in a third condition, the high-similarity items were arranged into a rhyming pattern (i.e., A, B, C, A', B', $\mathrm{C}^{\prime}$ ). Differences between low and high similarity were expected to be greater under conditions of varied relative to constant starting position. This finding would replicate the Nelson et al (1970) results using six-instead of eight-item lists. To the extent that rhyming pattern can also serve as an effective extrinsic cue, performance in the high-similarity pattern condition should be superior to that obtained when the highly similar words fail to form a pattern. The effectiveness of the pattern variable, however, may depend upon the constancy of the temporal position cues. Varying starting position may reduce the salience and hence the effectiveness of pattern, since recall will not begin on the initial item of the pattern sequence on every trial (e.g., B, C, $A^{\prime}, B^{\prime}, C^{\prime}$, A). However, since the pattern will be maintained in the sequence of the items, it may actually differentially facilitate recall when starting position is varied, since this procedure eliminates temporal constancy as a potential extrinsic cue.

\section{METHOD}

There were three conditions of similarity, two different word lists for each condition, and two starting position treatments, so that the experimental design conformed to a 3 by 2 by 2 factorial. Each $\mathrm{S}$ acquired a single list of six words in an individual session. The low-similarity lists were GAS, RUT, TEA, FIN, SKI, OWL and HUM, BAY, LEG, SHY, FIT, JOB. The high-similarity pattern lists were HAM, HUM, HAT, RAM, RUM, RAT and BAG, BEG, BED, LAG, LEG, LED. The high-similarity lists contained the same set of words, arranged so that no pattern was formed: RUM, HAT, HAM, RAT, HUM, RAM and LAG, BED, BEG, LED, BAG, LEG.

Practice was by the method of serial anticipation to a criterion of either two consecutive trials without error or 50 trials, whichever came first. Stimuli were pronounced but guessing was not required, so that $S$ could remain silent. In the constant starting position conditions, the anticipation sequence always started on the same item on successive trials. In the varied conditions, the starting position varied randomly from trial to trial. There was a 4-sec interval following each presentation of the list, after which the last item of the preceding trial was shown. Using the last item as the anticipation cue required learning six associations in each starting position condition.

For the low- and high-similarity treatments, each word in the above lists could appear as the initial item in the sequence, as determined by the random selection of one of the rows of a standard 6 by 6 Latin square. For example, if the words of these lists were consecutively numbered from 1 through 6 , one $S$ in the constant starting position condition may have learned 4,5 , $6,1,2,3$, and another $S$ may have acquired $3,4,5,6,1$, 2, etc. Thus, in the constant conditions, the initiating item was selected 


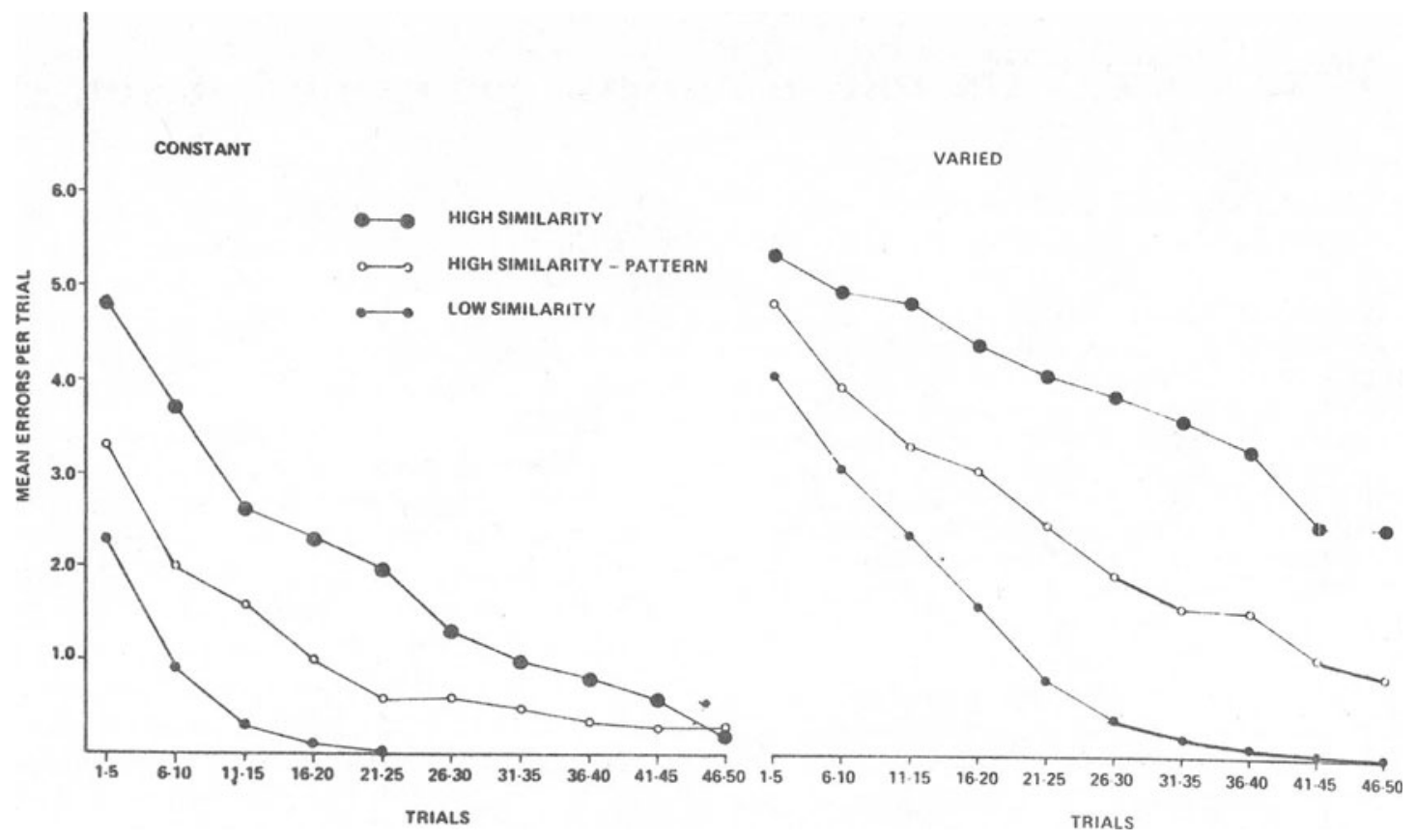

Fig. 1. Mean errors per trial as a function of starting position and similarity condition.

randomly for each $\mathrm{S}$ but, once selected, it remained constant from trial to trial. In the varied conditions, the initiating item varied randomly from $S$ to $S$ and from trial to trial. The only restriction was that each of the six sequences formed by the 6 by 6 Latin square was used once every six trials. For the high-similarity pattern lists, only two sequences were used in the constant starting position conditions (i.e., either 1, 2, 3, 4, 5, 6 or $4,5,6,1,2,3)$. In the varied starting position conditions, practice on the high-similarity pattern lists was initiated as in all other varied conditions. Thus, the rhyming pattern was preserved only by item sequence, not by ordinal position. However, within each block of six trials, the two sequences used for the constant conditions were each presented once.

Each word was typed in uppercase letters and was presented via a Kodak carousel slide projector, driven by an auxiliary timer at a 2 -sec rate. To insure understanding of the instructions, all Ss learned a practice list consisting of an alphabetic sequence (A, B, $\mathrm{C} \ldots \mathrm{K})$ before undertaking the experimental task. Starting position procedure in the practice task was consistent with that used in the experimental task.

The 96 Ss were students of introductory psychology who received points toward their grade for participation. The Ss were assigned to conditions in blocks of 12 , with one $S$ per condition in each block. Within each block, the order of conditions was determined by a table of random numbers. This procedure resulted in a total of 16 different Ss in each similarity/starting position condition. Each of two female Es tested half of the Ss for each condition.

\section{RESULTS}

Figure 1 presents mean errors per trial (omission plus comission) as a function of the principal conditions of the experiment, plotted in blocks of five trials. Comparison of these curves indicated that difficulty of acquisition was greater when starting position was varied than when constant and that difficulty generally increased in the order low similarity, high-similarity pattern, and high similarity. The analysis of variance of these data indicated that the main effects of starting position, $F(1,90)=38.52$, similarity condition, $\mathrm{F}(2,90)=27.20$, and trials, $\mathrm{F}(9,810)=148.94$, were all significant at $p<.01$. In addition, the interactions of Starting Position by Trials, $F(9,810)=3.68, p<.01$, Similarity Condition by Trials, $F(18,310)=1.91$, $\mathrm{p}<.05$, and Starting Position by Similarity Condition by Trials, $F(18,810)=4.52, p<.01$, were also reliable. As indicated in Fig. 1, eliminating the temporal constancy cues increased the differences between the similarity conditions, particularly toward the later portion of acquisition. By the sixth trial block (Trials 26-30), the interfering effects produced by similarity were much greater when these cues were eliminated relative to when they were left intact. The significant three-way interaction reflected this effect. Thus, the trial by trial differences between low and high similarity were greater when starting position was varied relative to when it was constant. This conclusion was reinforced by the results of an analysis of variance performed on only these data (i.e., pattern conditions omitted). The interactions of Similarity by Starting Position, $F(1,60)=4.52, p<.05$, and Similarity by Starting Position by Trials, $F(9,540)=8.10, p<.01$, were significant. Furthermore, the trial by trial differences between high-similarity pattern and high similarity were also greater when starting position was varied compared to when it was constant. This difference appeared to increase with increasing amounts of practice. The results of the separate statistical analysis of the high-similarity conditions (i.e., low-similarity conditions omitted) indicated that Pattern by Starting Position by Trials, $\mathrm{F}(9,540)=3.55, \mathrm{p}<.01$, was a 
reliable source of variance. Thus, when starting position was varied from trial to trial, recall was differentially sensitive to the rhyming pattern preserved within the sequence of words. A plot of the serial position curves for the lists containing the rhyming pattern suggested that the principal source of facilitation in these conditions was derived from organizing the lists into two units. Within each starting position condition, mean errors on Positions 1-3 and on Positions 4-6 were displayed as two V-shaped curves.

\section{DISCUSSION}

The results of the present experiment corroborate and extend those reported by Nelson et al (1970). Reliance upon features extrinsic to the words (temporal constancy cues) was greater when the items were similar relative to when they were distinct. This inference is based on the finding that the differences between low and high similarity are greater when starting position is varied from trial to trial than when it is constant on successive trials. The results of manipulations of rhyming pattern suggest that this factor can also serve as a functionally effective extrinsic cue. Arranging similar items into a rhyming pattern facilitates recall when the temporal cues are either constant or variable. Examination of the serial position curves associated with the pattern conditions suggests that this variable enhances recall by organizing the list into two separate but phonetically related units. The identity of the phonetic structure of the units may have served to facilitate the acquisition of the relative positions of the items (cf. Voss, 1968). This interpretation assumes that learning relative position is more easily accomplished when the list can be grouped into two parts compared to when relative position information encompasses all of the items within the list. This organizational process was apparently not totally disrupted by variations in starting position. In fact, the differences between the high-similarity pattern and high-similarity lists are greater when starting position is varied than when it is constant, especially toward the later portion of acquisition. Thus, the relative magnitude of the facilitation attributable to rhyming pattern is greater when the temporal constancy cues have been eliminated as potential extrinsic cues.

\section{REFERENCES}

Anisfeld, M., \& Knapp, M. Association, synonymity, and directionality in false recognition. Journal of Experimental Psychology, 1968, 77, 171-179.

Nelson, D. L. Words as sets of features: The role of phonological attributes. In R. F. Thompson and J. F. Voss (Eds.), Topics in learning and performance. New York: Academic Press, 1972.

Nelson, D. L., Wheeler, J., \& Bercov, S. Variations in item availability and distinctiveness and the role of temporal constancy cues in serial anticipation. Journal of Experimental Psychology, 1970, 86, 463-464.

Underwood, B. J. Attributes of memory. Psychological Review, 1969, 76, 559-573.

Voss, J. F. Serial acquisition as a function of number of successively occurring list items. Journal of Experimental Psychology, 1968, 78, 456-462.

Wickens, D. D. Encoding categories of words: An empirical approach to meaning. Psychological Review, 1970, 77, 1-15.

(Received for publication January 8, 1973.) 